\title{
Congruence properties of self-contained balanced sets
}

\author{
By Hanstaj GUpta.
}

(Received 10th June, 1938. Read 4th November, 1938.)

1. Let the set $b_{1}, b_{2}, b_{3}, \ldots, b_{k}$; of $k$ non-negative integers be denoted by $B_{k}$. Let $\xi B_{k}$ denote the set $\xi b_{1}, \xi b_{2}, \xi b_{3}, \ldots, \xi b_{k} ; \xi$ being any integer $>1$. Without loss of generality, we can suppose that $b \leqq b_{i+1}$.

In what follows, $G_{r}\left(B_{k}\right)$ denotes the sum of the products taken $r$ at a time of the members of set $B_{k} ; 0 \leqq r \leqq k$. We take $G_{0}\left(B_{k}\right)=1$. $S_{r}\left(B_{k}\right)$ stands for the sum of the $r$-th powers of the members of set $B_{k}$. All small letters denote integers $\geqq 0$, unless stated otherwise; $p$ 's denote primes $\geqq 2 ; m>2$; and $\phi$ is Euler's function.

If $m \equiv 0\left(\bmod p^{u}\right)$, but $\neq 0\left(\bmod p^{u+1}\right), u>0$, then we say that $m$ is $u$-potent in $p$, and write $\operatorname{Pot}_{p} m=u$. In the same way, when $\phi\left(p^{a}\right) \mid g$, but $\phi\left(p^{\alpha+1}\right)$ does not divide $g, \alpha>0$, then we say that $g$ is $\alpha$-piquant in $p$, and write $\operatorname{Piq}_{p} g=\alpha$. Evidently if $\operatorname{Piq}_{p} g=a$, then $\operatorname{Pot}_{p} g=a-1$, but not conversely. Again if $\operatorname{Piq}_{p} g=\alpha>0$, then we write $M_{g}$ for the product $\Pi\left(p^{a+[2 / p]}\right)$, and $N_{g}$ for the product $\prod_{0}\left(p^{1+[2 / p]}\right)$. Thus $N_{6}=7.3 .2^{2}$, and $M_{6}=7.3^{2} \cdot 2^{3}$.

A set $B_{k}$ is said to be self-contained modulo $m$, when for every number $\xi$ less than and prime to $m$, the members of set $\xi B_{k}$ are congruent modulo $m$ to the members of set $B_{k}$, each to each, though not necessarily in the same order.

If the members of set $B_{k}$ satisfy the relation:

$$
b_{i}+b_{k-i+1}=m, \quad i=1,2,3, \ldots, k ;
$$

then $B_{k}$ is called a balanced $m$-set.

2. We make use of the following

Lemma. If $\operatorname{Piq}_{p} g=\alpha$, then numbers $\xi$ prime to $p$, exist such that $\operatorname{Pot}_{p}\left(\xi^{g}-1\right)=a+\left[\frac{2}{p}\right]$.

Numbers $\xi$ exist such that $\operatorname{Pot}_{p}\left(\xi^{h}-1\right)=a+\left[\frac{2}{p}\right]$, where $h=\phi\left(p^{\alpha}\right)$. Let $g=s . h$, where $p$ does not divide $s$. Then since

$$
\left(\xi^{g}-1\right) /\left(\xi^{h}-1\right)=1+\xi^{h}+\xi^{2 h}+\ldots+\xi^{(s-1) h} \equiv s\left(\bmod p^{\alpha+[2 / p]}\right),
$$


we have

$$
\operatorname{Pot}_{p}\left(\xi^{g}-1\right)=\operatorname{Pot}_{p}\left(\xi^{h}-1\right)=a+\left[\frac{2}{p}\right] .
$$

3. Theorem 1. If $B_{k}$ be a balanced $m$-set, then

$$
2 G_{2 j+1}\left(B_{k}\right) \equiv(k-2 j) m G_{2 j}\left(B_{k}\right)\left(\bmod m^{2} n\right)
$$

where $n=m / 2$, only when $m$ is even and $k$ odd; otherwise $n=m$.

We have

Therefore

$$
\prod_{i=1}^{k}\left(x+b_{i}\right)=\prod_{i=1}^{k}\left(x+m-b_{i}\right) .
$$

$$
\sum_{r=0}^{k} G_{r}\left(B_{k}\right) x^{k-r}=\sum_{r=0}^{k}(-1)^{r} G_{r}\left(B_{k}\right)(x+m)^{k-r} .
$$

Equating the coefficients of $x^{k-(2 j+1)}$, we get

$$
\begin{gathered}
2 G_{2 j+1}\left(B_{k}\right)=\left(\begin{array}{c}
k-2 j \\
1
\end{array}\right) m G_{2 j}\left(B_{k}\right)-\left(\begin{array}{c}
k-2 j+1 \\
2
\end{array}\right) m^{2} G_{2 j-1}\left(B_{k}\right)+\ldots \\
+\left(\begin{array}{c}
k \\
2 j+1
\end{array}\right) m^{2 j+1} G_{0}\left(B_{k}\right) .
\end{gathered}
$$

"Therefore

$$
G_{2 j+1}\left(B_{k}\right) \equiv 0(\bmod n), j=0,1,2, \ldots,\left[\frac{k-1}{2}\right] .
$$

In particular

Hence the theorem.

$$
G_{2 j-1}\left(B_{k}\right) \equiv 0(\bmod n), j=1,2,3, \ldots,\left[\frac{k+1}{2}\right] .
$$

Again, if $B_{k}$ be a balanced $m$-set, we notice that

Therefore

$$
2 S_{2 j+1}\left(B_{k}\right)=\sum_{i=1}^{k}\left\{b_{i}^{2 j+1}+\left(m-b_{i}\right)^{2 j+1}\right\} .
$$

$$
2 S_{2 j+1}\left(B_{k}\right) \equiv(2 j+1) m S_{2 j}\left(B_{k}\right)\left(\bmod m^{2} t\right),
$$

where $t=m$ or $m / 2$, according as $m$ is odd or even.

4. Theorem 2. If $\boldsymbol{B}_{k}$ be a self-contained set modulo $m$, then

$$
G_{2 j}\left(B_{k}\right) \equiv 0 \equiv S_{2 j}\left(B_{k}\right)\left(\bmod p^{\eta-\alpha-[2 / p]}\right) ;
$$

where $\operatorname{Pot}_{p} m=u$, and $\operatorname{Piq}_{p}(2 j)=\alpha$; and $j>0$.

Evidently, we can suppose that $a \leqq u-\left[\frac{2}{p}\right]$. Now let $\xi$ be a number such that $\operatorname{Pot}_{p}\left(\xi^{2 j}-1\right)=\alpha+\left[\frac{2}{p}\right]$. 
Then $\quad \xi^{2 j} G_{2 j}\left(B_{k}\right)=G_{2 j}\left(\xi B_{k}\right) \equiv G_{2 j}\left(B_{k}\right)\left(\bmod p^{u}\right)$,

whence $\quad\left(\xi^{2 j}-1\right) G_{2 j}\left(B_{k}\right) \equiv 0\left(\bmod p^{u}\right)$,

and

$G_{2 j}\left(B_{k}\right) \equiv 0\left(\bmod p^{u-a-[2 / p]}\right)$.

As an immediate consequence of Theorem 2, we have

$$
\left(m, M_{2 j}\right) G_{2 j}\left(B_{k}\right) \equiv 0(\bmod m) \text {. }
$$

The above proof holds good also when $G$ is replaced by $S$.

5. Theorem 3. If $B_{k}$ be a balanced m-set, self-contained modulo $m$, then

$$
2\left(m, M_{2 j}\right) S_{2 j+1}\left(B_{k}\right) \equiv 0 \equiv 2\left(m, M_{2 j}\right) G_{2 j+1}\left(B_{k}\right)\left(\bmod m^{2}\right) ; j>0 .
$$

This theorem follows directly from theorems 1 and 2.

If $(k, 2 j)=g$, then the second part of this theorem takes the form

$$
2\left(m, M_{2 j} N_{g} / M_{g}\right) G_{2 j+1}\left(B_{k}\right) \equiv 0\left(\bmod m^{2}\right),
$$

for $\operatorname{Pot}_{p}(k-2 j) \geqq \operatorname{Pot}_{p} g$.

6. When set $B_{k}$ consists of integers less than and prime to $m$. Theorem 3 leads to the following generalisation of Leudesdorf's Theorem :

$$
2\left(m, N_{g}\right) G_{2 j+1}\left(B_{k}\right) \equiv 0\left(\bmod m^{2}\right) ; j \geqq 1, g=(k, 2 j), k=\phi(m) .
$$

Let $C$ denote the set of integers $1,2,3, \ldots, m-1$; and let $D$ denote the set $0,1,2,3, \ldots, m-1$.

Then for $j \geqq 1$, we have

and

$$
2\left(m, M_{2 j}\right) G_{2 j \div 1}(C) \equiv 0\left(\bmod m^{2}\right),
$$

$$
2\left(m-1, M_{2 j}\right) G_{2 j+1}(D) \equiv 0\left(\bmod (m-1)^{2}\right) .
$$

Since $G_{2 j+1}(C)=G_{2 j+1}(D)$, we get

$$
2\left(m .(m-1), M_{2 j}\right) G_{2 j+1}(C) \equiv 0\left(\bmod m^{2}(m-1)^{2}\right) .
$$

This is a generalisation ${ }^{1}$ of two theorems of Glaisher. The theorem holds when $G$ is replaced by $S$.

In particular, if $E$ denote the set of integers $1,2,3, \ldots, p^{u}-1$, we have

$$
G_{2 j+1}(E) \equiv 0\left(\bmod p^{2 u-\lambda-[2 / p]}\right),
$$

where $\lambda=\operatorname{Piq}_{p}(2 j)$, and $j \geqq 1$.

1 J. W. L. Glaisher, Messenger of Math., 28(1898), 184-185. For unother generalisation see H. Gupta, Proc. Edinburgh Math. Soc. (2), 4 (1935), 61-66.

Government College,

HoshIARPUR, INDIA. 